\title{
Targeting MYC: is it getting any easier?
}

\section{"....in tumor cells MYC is deregulated as result of constitutive activation of upstream oncogenic signaling pathways, or direct mutation in MYC genes."}

First draft submitted: 2 June 2016; Accepted for publication: 12 June 2016; Published online: 21 September 2016

Keywords: anticancer therapy $\bullet$ MYC $\bullet$ protein-protein interactions

The MYC bHLHZip transcription factor is a master regulator of cell growth, cell-cycle progression, metabolism and survival, which plays a central role in most human cancers [1-3].

MYC expression in normal cells is tightly regulated, while in tumor cells MYC is deregulated as result of constitutive activation of upstream oncogenic signaling pathways, or direct mutation in $M Y C$ genes. In vivo data have shown that inactivation of MYC dramatically halts tumor cell growth and proliferation, without invoking tumor escape pathways [4,5]. Somatic cells tolerate MYC inactivation over extended periods, and its effects are rapidly and completely reversible. Although MYC has become such an attractive therapeutic target no MYC inhibitors are yet in clinical studies.

\section{Direct inhibition}

MYC functions are tightly regulated by protein-protein interactions (PPIs) with a number of co-factors, and therefore targeting key PPIs has emerged as one of the most valuable approaches to downregulate its activity [6]. However, inhibition of PPIs with small molecules has been challenging as they associate through extensive interfaces via large surface areas often noncontiguous and with a lack of deep pockets. This presents challenges for assay development, biophysical and structural characterization of the ligand mode-of-action, and the optimization of the absorption, distribution, metabolism, and excretion (ADME) properties of the PPI inhibitors, as they tend to be rather large and hydrophobic compounds [7]. A structural feature that makes MYC even more different than a traditional molecular target is its intrinsically disordered nature. As an intrinsically disordered protein (IDP) MYC in the native state is disordered for its full length, and become ordered upon binding to another protein. MYC, thus, presents an exceptional degree of plasticity, and its PPIs are driven by a complex process of coupled folding and binding [6]. This could offer though an opportunity to develop small molecules that can induce a binding site (i.e., an extreme example of induced fit). Upon binding the compound would induce a conformational change in MYC that could result in the halting, or in the modulation, of its functions. A significant binding energy is however needed to overcome the high entropic cost. Furthermore, the compounds are more likely to bind in multiple states, which makes establishing modes of molecular recognition more difficult. Mainly for these reasons progress in developing direct MYC inhibitors has been slow.

The formation of the MYC:MAX complex is required for most if not all of MYC functions, and to date this PPI has been viewed as the most promising target for direct MYC inhibition. Molecules have been reported in the literature as putative MYC ligands that in vitro inhibit the MYC:MAX interaction [8]. Unfortunately, these compounds do not possess amenable pharmacokinetics and pharmacodynamics properties for in vivo application, and the characterization of their mode of bind-

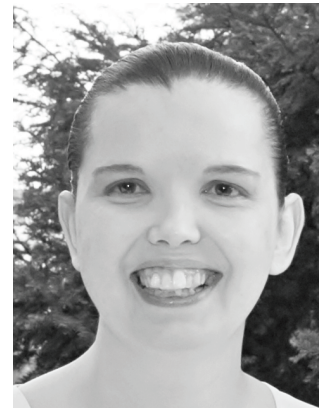

Giovanna Zinzalla Karolinska Institutet - Microbiology, Tumor \& Cell Biology, Nobels väg 16 Stockholm 171 77, Sweden giovanna.zinzalla@ki.se 
ing is very limited. It is important to highlight that the interaction of MYC with MAX involves two IDPs that undergo a coupled folding and binding process to form the complex. It is vital for the development of inhibitors to have a proper understanding of this process and carry out detailed biophysical studies to characterize the dynamic and plastic nature of the complex. We also require improving the biochemistry for developing new and more robust screening methodologies, and find better ways to characterize the mode of molecular binding of inhibitors. It is also critical to point out that this unconventional class of molecular targets requires the screening of chemical libraries more complex and covering a larger chemical space than those used for conventional targets (e.g., kinases). The use of innovative chemistry is also required when developing PPI inhibitors.

\section{"Transcription of MYC-target genes also depends on the activity of transcriptional kinases."}

Beyond the MYC:MAX interface, other PPIs could be explored as potential targets. The MYC C-terminal domain is not solely involved in forming the complex with MAX and binding to DNA, but it also recruits co-factors that regulate the MYC activity, such as INI1/hSNF5 and MIZ-1 [9]. As these PPIs involve MYC in complex with MAX (i.e., MYC is in a folded state), their inhibition could be more chemically tractable. Furthermore, this approach would allow stopping only selected MYC functions, rather than abolishing all of the MYC activities, potentially resulting in fewer side effects. Functional studies also highlighted the MBII region within MYC N-terminus is a potential target, due to its well-established role in recruiting co-factors such as TRAPP. Unfortunately, this region is also fully intrinsically disordered and there is limited biochemical and no structural information available about its PPIs.

\section{Indirect inhibition}

Because MYC has been deemed 'undruggable' a plethora of approaches has been explored aimed at finding conventional molecular targets that indirectly inhibit MYC.

\section{Targeting upstream}

Numerous upstream signal transduction pathways activate the transcription of the $M Y C$ gene. Targeting these pathways has been explored as a strategy to halt its activation [1], however, the ability of tumor cells to find escape pathways to maintain MYC activation is a serious drawback to this approach.

\section{Stopping MYC being made}

BRD4, a BET bromodomain-containing protein, acts as a nodal point for many of the upstream acti- vation-pathways. BRD4 binds acetylated histones via its bromodomain and stimulates $M Y C$ transcription. Numerous inhibitors of BRD bromodomain have shown great promise as anticancer agents [10]. Tumors can become resistant to these agents by activating $M Y C$ transcription via pathways that do not require BRD4, particularly via Wnt signaling. Nonetheless, as a limited number of alternative ways to directly activating $m y c$ transcription is available to cells, combination of BRD4 inhibitors with agents blocking rescue pathways could work.

\section{Stopping MYC working}

Transcription of MYC target genes requires a number of mediators that could be amenable to smallmolecule inhibition [11]. For example, MYC recruits the TRRAP complex that includes the histone acetyltransferases TIP60 and GCN5. Transcription of MYC-target genes also depends on the activity of transcriptional kinases, such as CDK-7, CDK-8 and CDK-9. Both these classes of enzymes are desirable for drug discovery and small-molecule inhibitors are being intensively pursued. However, the pleiotropic role of these enzymes could present serious drawbacks. Rather than going after the catalytic activity of these enzymes, the inhibition of the protein-protein interactions they make with MYC could offer more selectivity.

\section{MYC degradation}

An intriguing strategy focuses on modulating the stability of the MYC protein. The MYC proteins have a naturally short half-life, as they are efficiently targeted by the Ubiquitin Proteosome System (UPS). [12] The interactions between MYC proteins and the E3 ligases that target them are regulated by post-translational modifications such as phosphorylation and acetylation. Some serine and threonine phosphorylation prevent MYC degradation, and thus development of kinase inhibitors could be pursued as a way of enhancing MYC degradation. For example, approaches targeting the inhibition of MEK-ERK pathway, or PI3K-AKT signaling, are actively explored.

The Aurora A kinase binds to the N-terminus of MYC proteins to protect them from degradation by FBW7 E3 ligase. Allosteric inhibitors of this kinase that stop its binding to MYC, thereby allowing MYC degradation have shown promise as a way of targeting MYC [13]. An alternative strategy could be to inhibit the deubiquitinating enzymes (DUBs) that by removing poly-ubiquitin chains from MYC slow down its degradation. Several MYC-specific DUBs are overexpressed in cancer, and DUBs are attractive molecular targets for drug discovery. 


\section{Synthetic lethality}

Synthetic lethality-based approaches are being widely employed to indentify candidates for the development of anti-MYC drugs [14]. This strategy exploits the fact that cancer cells overexpressing MYC have unique dependencies that normal cells do not require. Inhibiting the pathways involved should selectively kill MYC-dependent cancer cells while leaving somatic cells largely unaffected (what is knows in genetics as a synthetic lethal interaction). Highly parallel RNAi screening has identified a multitude of these interactions, which could potentially offer many molecular targets for halting MYC-dependent tumor cells. A lot of attention has been focusing on kinases because of their tractability as molecular targets. For example, AMPK (AMP-dependent kinase), Cdk-1 and CSNK1E kinases have all been identified as critical for the survival of cells with high levels of MYC. A screen has also identified the ALDO A and PDK enzymes both of which are involved in metabolic pathways. Other hits from these screenings have produced some surprising MYC-dependencies such as the SAE1/2 SUMOactivating enzyme [15] and spliceosome core factors [16]. The jury is still out whether this strategy can lead to useful anticancer drugs. Success requires on the extent of how much MYC depends upon these putative tar-

\section{References}

1 McKeown MR, Bradner JE. Therapeutic strategies to inhibit MYC. Cold Spring Harb. Perspect. Med. 4(10), pii: a014266 (2014).

2 Dang CV. MYC on the path to cancer. Cell 149(1), 22-35 (2012).

3 Huang H, Weng H, Zhou H, Qu L. Attacking c-Myc: targeted and combined therapies for cancer. Curr. Pharm. Des. 20(42), 6543-6554 (2014).

4 Soucek L, Whitfield J, Martins CP et al. Modelling Myc inhibition as a cancer therapy. Nature 455(7213), 679-683 (2008).

5 Morton JP, Sansom OJ. MYC-y mice: from tumour initiation to therapeutic targeting of endogenous MYC. Mol. Oncol. 7(2), 248-258 (2013).

6 Sammak S, Zinzalla G. Targeting protein-protein interactions (PPIs) of transcription factors: challenges of intrinsically disordered proteins (IDPs) and regions (IDRs). Prog. Biophys. Mol. Biol. 119(1), 41-46 (2015).

7 Laraia L, Mckenzie G, Spring DR, Venkitaraman AR, Huggins DJ. Overcoming chemical, biological, and computational challenges in the development of inhibitors targeting proteinprotein interactions. Chem. Biol. 22(6), 689-703 (2015).

8 Fletcher S, Prochownik EV. Small-molecule inhibitors of the MYC oncoprotein. Biochim. Biophys. Acta 1849(5), 525-543 (2015).

9 Tu WB, Helander S, Pilstal R et al. Myc and its interactors take shape. Biochim. Biophys. Acta 1849(5), 469-483 (2015). gets and how much the normal cells are not, and how easy such inter-dependency can be escaped by the cancer cells [17]. We will only know this once small-molecules inhibitors get tested at the very least in mouse modes of MYC-driven tumorgenesis, which can be an expensive and lengthy process.

Although indirect approaches to targeting MYC have provided some promising potential drugs (e.g., BRD4 inhibitors for leukemia) ultimately to obtain the most effective agents we will have to bite the bullet and directly inhibit MYC. This will need better ways of characterizing this protein and the interactions that it makes, drawing upon our growing knowledge of IDPs [18,19], and more imaginative ways of developing ligands that can modulate these interactions making use of recent developments in chemistry technologies [20-22].

\section{Financial \& competing interests disclosure}

The author has no relevant affiliations or financial involvement with any organization or entity with a financial interest in or financial conflict with the subject matter or materials discussed in the manuscript. This includes employment, consultancies, honoraria, stock ownership or options, expert testimony, grants or patents received or pending, or royalties.

No writing assistance was utilized in the production of this manuscript.

10 Delmore JE, Issa GC, Lemieux ME et al. BET bromodomain inhibition as a therapeutic strategy to target c-Myc. Cell 146(6), 904-917 (2011).

11 Posternak V, Cole MD. Strategically targeting MYC in cancer. F1000Research 5, pii:F1000 Faculty Rev-408 (2016).

12 Farrell AS, Sears RC. MYC degradation. Cold Spring Harb. Perspect. Med. 4(3), pii: a014365 (2014).

13 Gustafson WC, Meyerowitz JG, Nekritz EA et al. Drugging MYCN through an allosteric transition in Aurora kinase A. Cancer Cell 26(3), 414-427 (2014).

14 Li X, Zhang XA, Li X, Xie W, Huang S. MYC-mediated synthetic lethality for treating tumors. Curr. Cancer Drug Targets 15(2), 99-115 (2015).

15 Kessler JD, Kahle KT, Sun T et al. A SUMOylationdependent transcriptional subprogram is required for Mycdriven tumorigenesis. Science 335(6066), 348-353 (2012).

16 Hsu TY, Simon LM, Neill NJ et al. The spliceosome is a therapeutic vulnerability in MYC-driven cancer. Nature 525(7569), 384-388 (2015).

17 Evan G. Cancer. Taking a back door to target Myc. Science 335(6066), 293-294 (2012).

18 Gianni S, Dogan J, Jemth P. Coupled binding and folding of intrinsically disordered proteins: what can we learn from kinetics? Curr. Opin. Struct. Biol. 36, 18-24 (2016).

19 Zhou M, Li Q, Wang R. Current experimental methods for characterizing protein-protein interactions. ChemMedChem 11(8), 738-756 (2016). 
20 Kuenemann MA, Sperandio O, Labbé CM, Lagorce D, Miteva MA, Villoutreix BO. In silico design of low molecular weight protein-protein interaction inhibitors: overall concept and recent advances. Prog. Biophys. Mol. Biol. 119(1), 20-32 (2015).

21 Fischer G, Rossmann M, Hyvönen M. Alternative modulation of protein-protein interactions by small molecules. Curr. Opin. Biotechnol. 35, 78-85 (2015).
22 -Gimeno M, Glas A, Koch O, Grossmann TN. Structurebased design of inhibitors of protein-protein interactions: mimicking peptide binding epitopes. Angew. Chem. Int. Ed. 54(31), 8896-8927 (2015). 